\title{
miR-206 serves an important role in polycystic ovary syndrome through modulating ovarian granulosa cell proliferation and apoptosis
}

\author{
JIE ZHOU* ${ }^{*}$, XUEJING JIN*, ZHUMEI SHENG and ZHIFEN ZHANG \\ Reproductive Endocrine Center, Hangzhou Women's Hospital, Hangzhou, Zhejiang 310008, P.R. China
}

Received April 16, 2020; Accepted August 21, 2020

DOI: $10.3892 / \mathrm{etm} .2021 .9610$

\begin{abstract}
An increasing number of studies have reported that microRNAs (miRNAs) have an important role in polycystic ovary syndrome (PCOS). Downregulation of miR-206 in patients with PCOS has been found, however, its specific role remains unclear. The present study aimed to investigate the roles of miR-206 in (PCOS) and to determine the underlying molecular mechanisms. Reverse transcription-quantitative PCR (RT-qPCR) was performed to analyze the expression levels of miR-206 in normal ovarian surface epithelial IOSE80 cells and human ovarian granulosa cell-like KGN cells. TargetScan was used to predict the target gene of miR-206, which was subsequently verified using a dual-luciferase reporter gene assay. The mRNA expression levels of cyclin D2 (CCND2) and the transfection efficiencies of the miR-206 mimic and CCDN2 overexpression plasmid were determined using RT-qPCR analysis. The protein expression levels of CCND2, cleaved-caspase-3 and pro-caspase-3 were analyzed using western blotting, and an MTT assay and flow cytometric analysis were used to evaluate the cell viability and levels of apoptosis, respectively, in the cells following transfection. Finally, the activity of caspase- 3 was analyzed using a caspase- 3 activity assay kit. The results of the present study revealed that the expression levels of miR-206 were downregulated in KGN cells compared with IOSE80 cells. CCND2 was predicted and verified to be a direct target gene of miR-206, and the mRNA and protein expression levels of CCND2 were discovered to be upregulated in KGN cells compared with IOSE80 cells. The miR-206 mimic and CCND2 overexpression plasmid significantly upregulated the expression levels of miR-206 and CCND2, respectively, in KGN cells. The
\end{abstract}

Correspondence to: Dr Zhifen Zhang, Reproductive Endocrine Center, Hangzhou Women's Hospital, 369 Kunpeng Road, Hangzhou, Zhejiang 310008, P.R. China

E-mail: ff56745@163.com

*Contributed equally

Key words: microRNA-206, cyclin D2, polycystic ovary syndrome, ovarian granulosa cells
miR-206 mimic also downregulated the expression levels of CCND2 in KGN cells, while this effect was reversed following the transfection with the CCND2 overexpression plasmid. Compared with the mimic control group, the miR-206 mimic significantly decreased the cell viability, induced the levels of apoptosis, increased the activity of caspase-3, upregulated cleaved-caspase-3 protein expression levels and downregulated pro-caspase-3 protein expression levels in KGN cells following transfection; these effects were reversed following the overexpression of CCND2. In conclusion, the findings of the present study suggested that miR-206 may serve an important role in PCOS through modulating ovarian granulosa cell viability and apoptosis.

\section{Introduction}

Polycystic ovary syndrome (PCOS), also known as Stein-Leventhal syndrome, is a series of symptoms caused by increased levels of male hormones in women, which include irregular menstruation, excessive menstruation, hirsutism, acne, pelvic pain, difficulty in conception, acanthosis nigricans and other symptoms (1-3). There are several conditions related to PCOS, such as obesity, obstructive sleep apnea, cardiovascular diseases, affective disorders and endometrial cancer (4). PCOS is affected by genetic and environmental factors; the risk factors include obesity, insufficient exercise or family history (5). Currently, there is no specific cure for PCOS (6,7). Therefore, the development of novel and effective treatment strategies for PCOS are required.

The ovary is an important organ for follicle development and ovulation, and the development of the follicle is accompanied by the growth, development and differentiation of granulosa cells $(8,9)$. The proliferation and apoptosis of ovarian granulosa cells was discovered to be closely associated with ovarian development $(10,11)$, thus, ovarian granulosa cells are usually used to study the associated mechanisms of PCOS (12-14). For this reason, the present study also studied PCOS using ovarian granulosa cells.

MicroRNAs (miRNAs/miRs) are a set of small non-coding RNAs of 20-22 nucleotides in length, which are involved in the growth of several types of cell and regulate the expression levels of target genes (15). An increasing number of studies have suggested that miRNAs may be associated with numerous types of human disease, including cancer, 
cardiovascular diseases, gynecological diseases and inflammatory diseases (16-19). In addition, previous studies have reported the roles of miRNAs in PCOS, such as miR-27a-3p and miR-518f-3p $(20,21)$. The mechanisms by which miRNAs are involved in the pathogenesis of PCOS are multifaceted and include their ability to affect follicular development or promote ovarian dysfunction, and to regulate ovarian hormones, and granulosa cell proliferation and apoptosis $(22,23)$. In previous studies, the expression levels of miR-206, which is located on human chromosome 6, were discovered to be downregulated in patients with PCOS; however, its specific role remains unclear (24).

The present study aimed to determine whether miR-206 served a significant role in patients with PCOS and further investigated its molecular mechanisms to provide a deeper theoretical basis and potential novel strategies for the treatment of PCOS.

\section{Materials and methods}

Cell culture. Normal ovarian surface epithelial IOSE80 cells were used as the control cells in the present study (25). IOSE80 cells and human ovarian granulosa cell-like KGN cells were obtained from the American Type Culture Collection. The cells were cultured in DMEM (Gibco; Thermo Fisher Scientifc, Inc.) supplemented with 10\% FBS (Gibco; Thermo Fisher Scientific, Inc.), and maintained at $37^{\circ} \mathrm{C}$ in a humidified atmosphere of $5 \% \mathrm{CO}_{2}$.

$R N A$ extraction and reverse transcription-quantitative PCR $(R T-q P C R)$. Total RNA was extracted from the cells using an PicoPure ${ }^{\mathrm{TM}}$ RNA Isolation kit (Invitrogen; Thermo Fisher Scientific, Inc.) and reverse transcribed into cDNA using a High Capacity cDNA Reverse Transcription Kit (Invitrogen; Thermo Fisher Scientific, Inc.). The following temperature conditions for RT were as follows: $70^{\circ} \mathrm{C}$ for $5 \mathrm{~min}, 37^{\circ} \mathrm{C}$ for $5 \mathrm{~min}$ and $42^{\circ} \mathrm{C}$ for $60 \mathrm{~min}$. qPCR was subsequently performed to quantify the expression levels of miRNA and mRNA on a Prism 7000 Real-Time PCR system (Applied Biosystems; Thermo Fisher Scientific, Inc.) using the SYBR qPCR Master Mix (Vazyme Biotech Co., Ltd.) according to the manufacturer's protocol. The primers were supplied by Sangon Biotech Co., Ltd. The following thermocycling conditions were used for the qPCR: Initial denaturation for $5 \mathrm{~min}$ at $95^{\circ} \mathrm{C}$; followed by 40 cycles of denaturation at $95^{\circ} \mathrm{C}$ for $10 \mathrm{sec}$, annealing at $60^{\circ} \mathrm{C}$ for $30 \mathrm{sec}$ and extension at $72^{\circ} \mathrm{C}$ for $34 \mathrm{sec}$. GAPDH was used as the internal loading control for mRNA expression levels, while U6 was used as the internal loading control for miR-206 expression levels. The primer sequences used for the PCR were listed as follows: GAPDH forward, 5'-CTTTGGTAT CGTGGAAGGACTC-3' and reverse, 5'-GTAGAGGCAGGG ATGATGTTCT-3'; U6 forward, 5'-GCTTCGGCAGCACAT ATACTAAAAT-3' and reverse, 5'-CGCTTCACGAATTTG CGTGTCAT-3'; miR-206 forward, 5'-GCGTCTGGAATG TAAGGAAGTG-3' and reverse, 5'-GTGCAGGGTCCGAGG T-3'; and CCND2 forward, 5'-TCCAAACTCAAAGAGACC AGC-3'; and reverse, 5'-TTCCACTTCAACTTCCCCAG-3'.

The relative mRNA expression levels of miR-206 and cyclin D2 (CCND2) were quantified using the $2^{-\Delta \Delta C q}$ method (26). All experiments were performed $\geq 3$ times.
Cell transfection. Following the overnight culture, KGN cells $\left(5 \times 10^{4}\right.$ cells per well) were transfected with the $100 \mathrm{nM}$ mimic control (5'-UUUGUACUACACAAAAGUACU G-3'; Shanghai GenePharma Co., Ltd.), 100 nM miR-206 mimic (5'-TGGAATGTAAGGAAGTGTGTGG-3'; Shanghai GenePharma Co., Ltd.), $1 \mu \mathrm{g}$ cyclin D2 CRISPR Activation Plasmid (CCND2-plasmid; cat. no. sc-401236-ACT; Santa Cruz Biotechnology, Inc.), $1 \mu \mathrm{g}$ Control CRISPR Activation Plasmid (control-plasmid; cat. no. sc-437275; Santa Cruz Biotechnology, Inc.), $100 \mathrm{nM}$ miR-206 mimic $+1 \mu \mathrm{g}$ control-plasmid or $100 \mathrm{nM}$ miR-206 mimic $+1 \mu \mathrm{g}$ CCND2-plasmid using Lipofectamine ${ }^{\circledR} 2000$ reagent (Invitrogen; Thermo Fisher Scientific, Inc.). Following transfection at $37^{\circ} \mathrm{C}$ for $48 \mathrm{~h}$, the transfection efficiency of the cells was analyzed using RT-qPCR. Cells without any treatment were used as the control.

miRNA target analysis and Dual-Luciferase reporter assay. The relationship between miR-206 and CCND2 was determined using TargetScan release 7.1 software (www.targetscan. org/vert_71). The 3'-untranslated region (UTR) of CCND2 containing the target sequence of miR-206 was obtained using RT-qPCR and cloned into a pmirGLO vector (Promega Corporation) to construct the wild-type (WT) reporter vector, CCND2-WT. Similarly, a mutant (MUT) vector, CCND2-MUT, was created. A QuikChange Site-Directed Mutagenesis kit (Stratagene; Agilent Technologies, Inc.) was applied according to the manufacturer's instructions to point-mutate the miR-206 binding domain in the 3'UTR of CCND2. Subsequently, 293 cells $\left(5 \times 10^{4}\right.$ cells per well) were cultured at $37^{\circ} \mathrm{C}$ for $24 \mathrm{~h}$ and then co-transfected with $1 \mathrm{ng}$ CCND2-WT or $1 \mathrm{ng}$ CCND2-MUT and the $100 \mathrm{nM}$ miR-206 mimic or $100 \mathrm{nM}$ mimic control using Lipofectamine 2000 reagent at $37^{\circ} \mathrm{C}$ for $48 \mathrm{~h}$. The relative luciferase activity was measured using a Dual-Luciferase reporter assay system (Promega Corporation), according to the manufacturer's protocol. The relative luciferase activity was normalized to Renilla luciferase activity. All the experiments were performed $\geq 3$ times.

Western blotting. Total protein was extracted from IOSE80 and KGN cells using RIPA Lysis Buffer (Beyotime Institute of Biotechnology). Total protein was quantified using a BCA protein assay kit (Pierce; Thermo Fisher Scientific, Inc.) and equal amounts (40 $\mu \mathrm{g}$ per lane) of protein were separated via $10 \%$ SDS-PAGE. The separated proteins were subsequently transferred onto PVDF membranes and blocked with PBS-(0.1\%) Tween-20 (PBST) solution containing 5\% non-fat milk at room temperature for $1 \mathrm{~h}$. The membranes were then incubated with the following primary antibodies at $4^{\circ} \mathrm{C}$ overnight: anti-CCND2 (cat. no. sc-376676; 1:1,000; Santa Cruz Biotechnology, Inc.), anti-cleaved-caspase-3 (cat. no. 9664; 1:1,000; Cell Signaling Technology), anti-pro-caspase-3 (cat. no. sc-373730; 1:1,000; Santa Cruz Biotechnology, Inc.) or anti-GAPDH (cat. no. sc-166574; 1:1,000; Santa Cruz Biotechnology, Inc.). Following the primary antibody incubation, the membranes were washed 3 times with PBST and incubated with the horseradish peroxidase-conjugated secondary antibodies (cat. nos. bs-0296G-HRP and bs-0295G-HRP; 1:500; BIOSS) for $40 \mathrm{~min}$ at room 
temperature. The protein bands were visualized using an ECL reagent (Pierce; Thermo Fisher Scientifc, Inc.), according to the manufacturer's instructions. All experiments were performed 3 times. ImageJ software (version 2.0; National Institutes of Health) was used to quantify the band intensity.

MTT assay. KGN cells ( $5 \times 10^{4}$ cells per well) were cultured in 96-well plates at $37^{\circ} \mathrm{C}$ for $24 \mathrm{~h}$, then the cells were transfected with $100 \mathrm{nM}$ mimic control, $100 \mathrm{nM}$ miR-206 mimic, $100 \mathrm{nM}$ miR-206 mimic $+1 \mu \mathrm{g}$ control-plasmid or $100 \mathrm{nM}$ miR-206 mimic $+1 \mu \mathrm{g}$ CCND2-plasmid for $48 \mathrm{~h}$. Subsequently, $10 \mu \mathrm{l}$ MTT solution (Beyotime Institute of Biotechnology) was added/well and incubated at $37^{\circ} \mathrm{C}$ for a further $4 \mathrm{~h}$. Subsequently, $100 \mu \mathrm{l}$ DMSO was added into each well at $37^{\circ} \mathrm{C}$ for $3 \mathrm{~h}$ to solubilize the formazan product after the culture medium was removed. The absorbance was measured at $570 \mathrm{~nm}$ using a microplate reader (Bio-Rad Laboratories, Inc.). The cell viability was calculated by normalizing each group with the control group using the optical density values.

Flow cytometric analysis of apoptosis. To analyze cell apoptosis, Annexin V/PI Apoptosis Detection kit (Beyotime Institute of Biotechnology) according to the manufacturer's protocol. KGN cells were collected by trypsinization through centrifugation $\left(1,000 \mathrm{x} \mathrm{g}, 5 \mathrm{~min}, 4^{\circ} \mathrm{C}\right)$, washed with $\mathrm{PBS}$ and then resuspended in $1 \mathrm{X}$ binding buffer at a density of $1 \times 10^{6}$ cells $/ \mathrm{ml}$. Subsequently, $100 \mu 1$ cell suspension was added into a $5 \mathrm{ml}$ tube, which was incubated with $5 \mu \mathrm{l}$ Annexin V-FITC and PI at room temperature for $20 \mathrm{~min}$, according to the manufacturers' protocols. The stained cells were analyzed using a BD FACSCalibur flow cytometer (BD Biosciences) and apoptotic rate (the percentage of early + late apoptotic cells) was calculated. Data were analyzed using CellQuest $^{\mathrm{TM}}$ software (version 5.1; BD Biosciences). All the experiments were performed $\geq 3$ times.

Detection of caspase- 3 activity. KGN cells $\left(5 \times 10^{4}\right.$ cells) were plated into 96 -well plates and incubated at $37^{\circ} \mathrm{C}$ overnight. Following the incubation, the cells were transfected for $48 \mathrm{~h}$, as described above, and then collected by trypsinization through centrifugation $\left(600 \mathrm{x} \mathrm{g} ; 4^{\circ} \mathrm{C} ; 5 \mathrm{~min}\right)$. Subsequently, the caspase-3 activity was immediately detected using a caspase-3 activity assay kit (Beyotime Institute of Biotechnology), according to the manufacturer's protocol. The absorbance was recorded at $405 \mathrm{~nm}$ using a microplate reader (Bio-Rad Laboratories, Inc.); caspase-3 activity was measured using optical density values.

Statistical analysis. Statistical analysis was performed using GraphPad Prism 6 software (GraphPad Software, Inc.) and data are presented as the mean \pm standard deviation of $\geq 3$ independent experiments. Statistical differences between groups were determined using an unpaired Student's t-test or one-way ANOVA followed by Tukey's post hoc test. $\mathrm{P}<0.05$ was considered to indicate a statistically significant difference.

\section{Results}

miR-206 expression levels are downregulated in KGN cells. The relative expression levels of miR-206 in normal ovarian

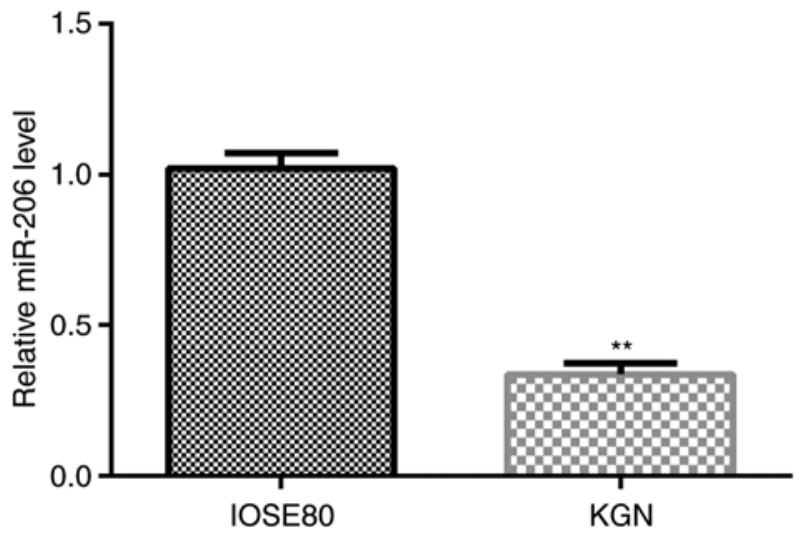

Figure 1. Expression levels of miR-206 in IOSE80 cells and KGN cells. Reverse transcription-quantitative PCR was performed to analyze the mRNA expression levels of miR-206 in IOSE80 cells and KGN cells. ${ }^{* * *} \mathrm{P}<0.01$ vs. IOSE80. miR, microRNA.

surface epithelial IOSE80 cells and human ovarian granulosa KGN cells were analyzed using RT-qPCR. As shown in Fig. 1, the expression levels of miR-206 were significantly downregulated in KGN cells compared with in IOSE80 cells.

CCND2 is a direct target gene of miR-206 and demonstrates upregulated expression levels in KGN cells. To investigate the molecular mechanisms of miR-206 in ovarian granulosa cells, the direct target gene of miR-206 was identified through the bioinformatics tool TargetScan. The predicted results indicated that CCND2 was a potential target of miR-206 (Fig. 2A). In addition, a Dual-Luciferase reporter assay was performed in 293 cells co-transfected with a luciferase vector plasmid carrying CCDND2-WT/MUT and the miR-206 mimic or mimic control to validate the predicted binding site of miR-206 on the CCND2 3'-UTR; the miR-206 mimic significantly decreased the relative luciferase activity of the CCND2-WT 3'-UTR compared with the mimic control and CCDN2-WT-transfected cells, whereas no significant differences were observed in the relative luciferase activity between the cells co-transfected with the CCND2-MUT 3'-UTR and the miR-206 mimic or mimic control (Fig. 2B). These results indicated that miR-206 may directly target CCND2. In addition, the mRNA and protein expression levels of CCND2 in IOSE80 cells and KGN cells were investigated. As shown in Fig. 2C-E, the mRNA and protein expression levels of CCND2 were significantly upregulated in KGN cells compared with IOSE80 cells.

miR-206 downregulates the expression levels of CCND2 in KGN cells. KGN cells were transfected with the mimic control, miR-206 mimic, CCND2-plasmid, or control-plasmid, and RT-qPCR was used to analyze the transfection efficiency. Compared with the mimic control group, the miR-206 mimic significantly upregulated the expression levels of miR-206 in KGN cells, while compared with the control-plasmid group, the CCND2-plasmid significantly upregulated CCND2 mRNA expression levels in KGN cells (Fig. 3A and B). In addition, the results of the co-transfection in KGN cells revealed that compared with the mimic control group, the miR-206 mimic downregulated the mRNA and protein expression levels 

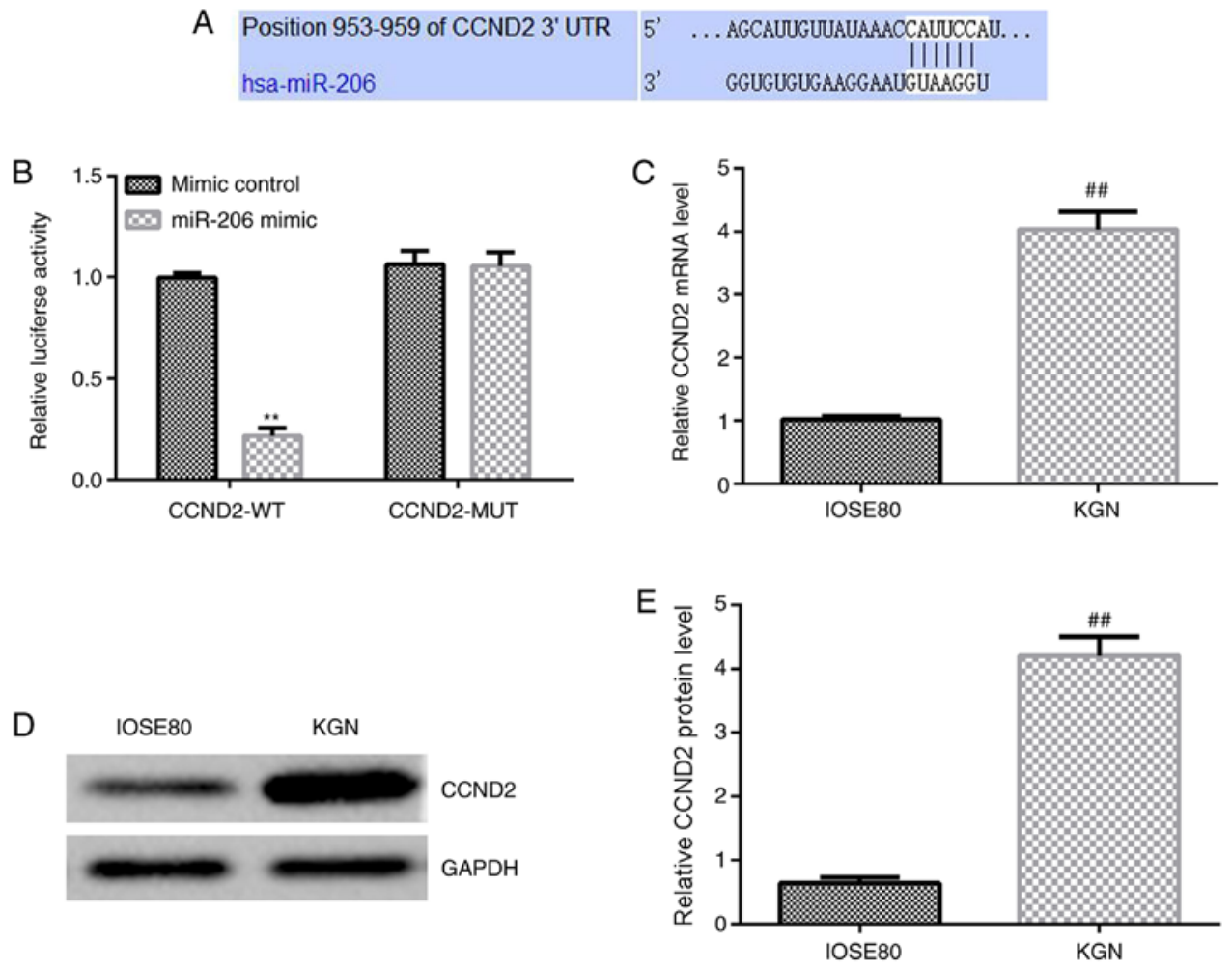

Figure 2. miR-206 directly targets CCND2. (A) Predicted WT miR-206 binding sites in the 3'-UTR of CCND2. (B) 293 cells were co-transfected with the CCND2-WT/MUT 3'-UTR luciferase reporter plasmid and the miR-206 mimic or mimic control. Following $48 \mathrm{~h}$ of transfection, the relative luciferase activity was analyzed using a Dual-Luciferase reporter assay. ${ }^{* * *} \mathrm{P}<0.01$ vs. mimic control group. (C) mRNA expression levels of CCND2 in IOSE80 and KGN cells were analyzed using reverse transcription-quantitative PCR. (D) Western blotting was performed to analyze the relative protein expression levels of CCND2 in IOSE80 and KGN cells. (E) Semi-quantification of CCND2 expression levels from part (D). ${ }^{\# \#} \mathrm{P}<0.01$ vs. IOSE80. miR, microRNA; CCND2, cyclin D2; WT, wild-type; UTR, untranslated region; MUT, mutant.

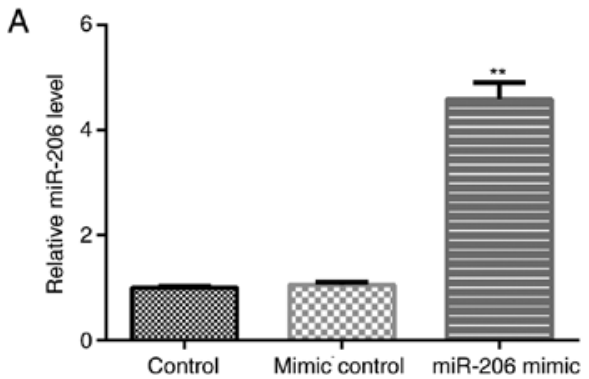

C

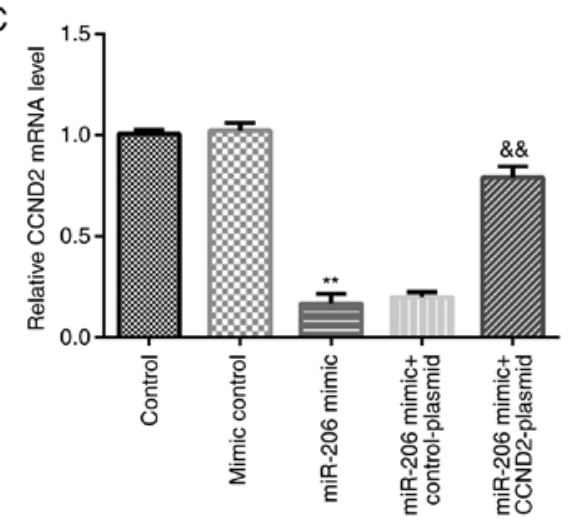

B

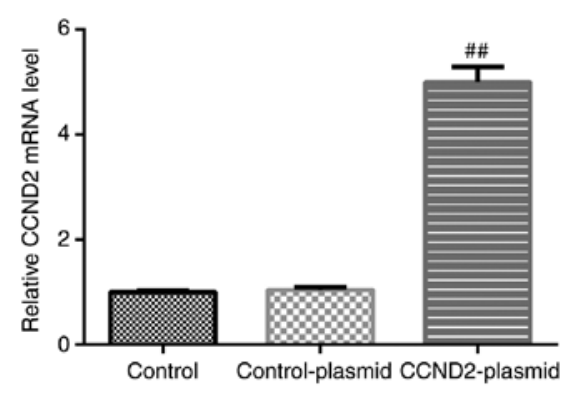

D

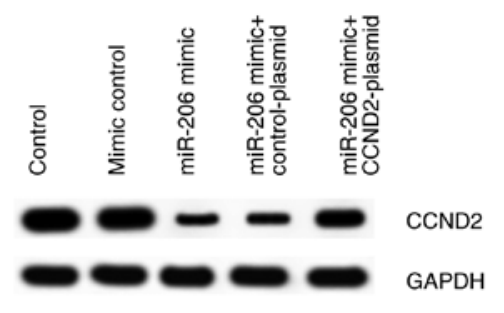

Figure 3. Transfection efficiency of the miR-206 mimic and CCND2-plasmid in KGN cells. (A) mRNA expression levels of miR-206 in KGN cells following the transfection with the miR-206 mimic or mimic control were analyzed using RT-qPCR. ${ }^{* *} \mathrm{P}<0.01 \mathrm{vs}$. mimic control group. (B) mRNA expression levels of CCND2 in KGN cells following the transfection with the control-plasmid or CCDN2-plasmid were analyzed using RT-qPCR. ${ }^{\# \#<0.01 ~ v s . ~ c o n t r o l-p l a s m i d ~}$ group. (C) mRNA expression levels of CCND2 in KGN cells following the different transfections were analyzed using RT-qPCR. ${ }^{* *} \mathrm{P}<0.01$ vs. mimic control group; \&\& $<<0.01$ vs. miR-206 mimic + control-plasmid group. (D) Protein expression levels of CCND2 in KGN cells following the different transfections were analyzed using western blotting. miR, microRNA; CCND2, cyclin D2; RT-qPCR, reverse transcription-quantitative PCR. 

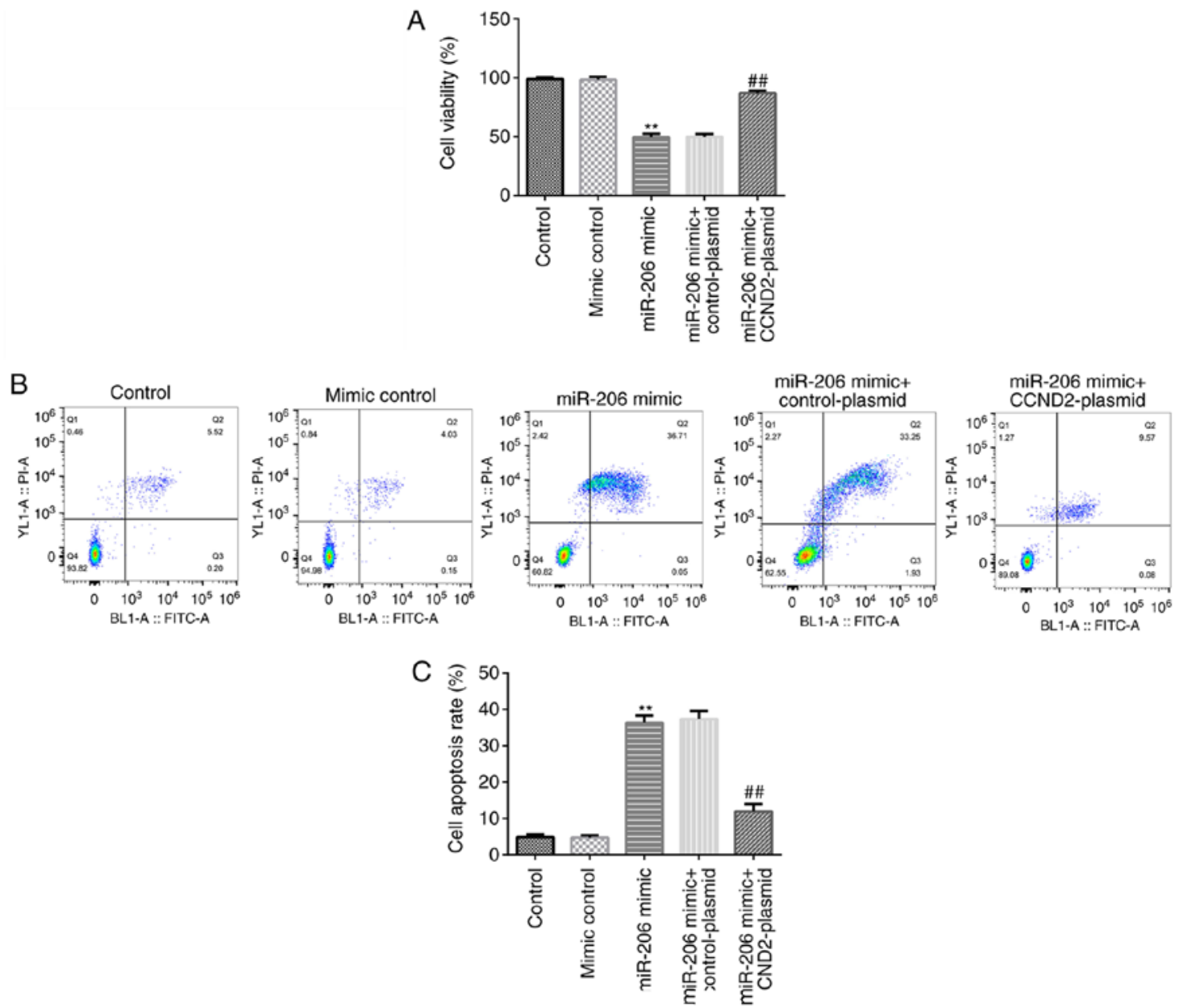

Figure 4. Effects of the miR-206 mimic and CCND2-plasmid on cell viability and apoptosis in KGN cells. (A) Cell viability of transfected KGN cells was analyzed using an MTT assay. (B) Flow cytometric analysis of apoptosis in transfected KGN cells. (C) Semi-quantification of the levels of cell apoptosis from part (B). ${ }^{* *} \mathrm{P}<0.01$ vs. mimic control group; ${ }^{\# \#} \mathrm{P}<0.01$ vs. miR-206 mimic + control-plasmid group. miR, microRNA; CCND2, cyclin D2; PI, propidium iodide.
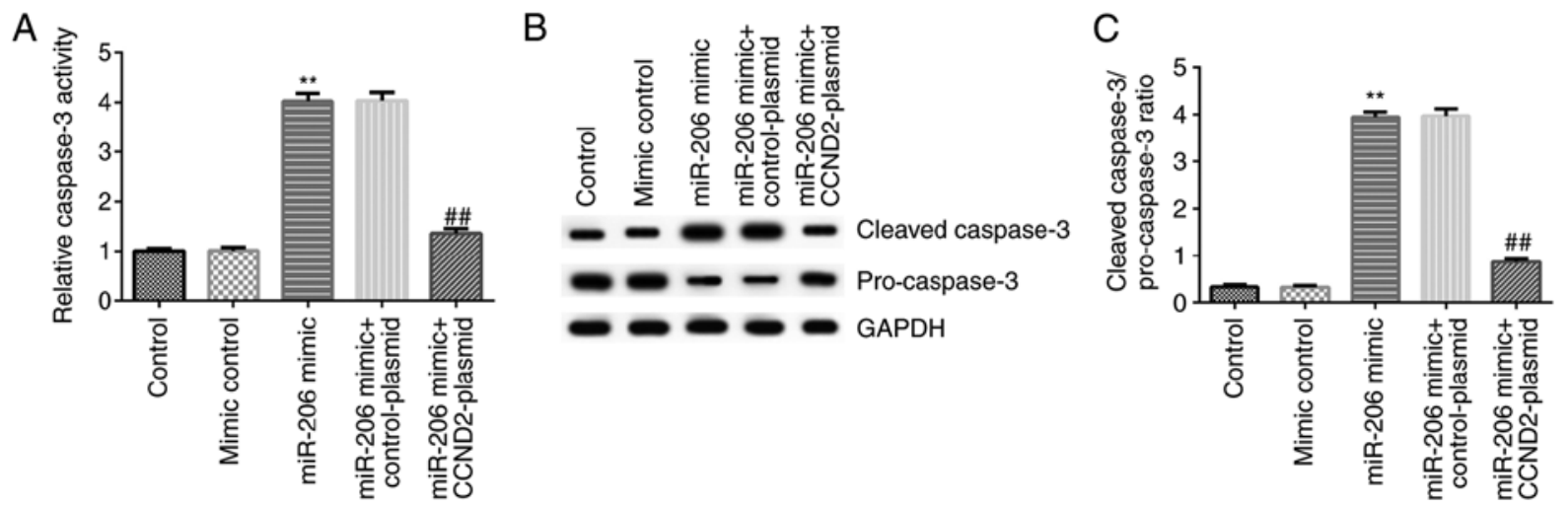

Figure 5. Effects of the miR-206 mimic and CCND2-plasmid on the activity and expression levels of caspase-3 in KGN cells. (A) Caspase-3 activity in KGN cells following the different transfections was detected using a caspase-3 activity assay kit. (B) Protein expression levels of cleaved-caspase-3 and pro-caspase-3 in transfected KGN cells were analyzed using western blot analysis. (C) Semi-quantification of the ratio of cleaved-caspase-3/pro-caspase-3 expression levels in transfected KGN cells from part $(\mathrm{B}) .{ }^{* *} \mathrm{P}<0.01$ vs. mimic control group; ${ }^{\# \#} \mathrm{P}<0.01$ vs. miR-206 mimic + control-plasmid group. miR, microRNA; CCND2, cyclin D2.

of CCND2 in KGN cells; however, this reduction could be reversed by the co-transfection with the CCND2-plasmid (Fig. 3C and D).
miR-206 inhibits cell viability and induces cell apoptosis in $K G N$ cells by targeting CCND2. An MTT assay and flow cytometric analysis were performed to determine the cell viability 
and levels of apoptosis. As shown in Fig. 4A-C, compared with the mimic control, the miR-206 mimic significantly reduced the cell viability and induced apoptosis in KGN cells, while these effects were significantly reversed following the co-transfection with the CCND2-plasmid.

miR-206 induces the apoptosis of KGN cells by regulating caspase-3 activity. To investigate the potential mechanism of miR-206 in the regulation of apoptosis in KGN cells, the activity and related protein expression levels of caspase-3 were analyzed in KGN cells following cell transfection. The results revealed that compared with the mimic control, the miR-206 mimic significantly increased the activity of caspase- 3 , and upregulated the protein expression levels of cleaved-caspase-3 and the cleaved-caspase-3/pro-caspase-3 ratio, while downregulating the protein expression levels of pro-caspase-3, in KGN cells. All of these effects were significantly reversed following the co-transfection with the CCND2-plasmid (Fig. 5A-C).

\section{Discussion}

PCOS is one of the most common types of endocrine disease among women aged 18-44 years (4). In fact, it has been suggested that the incidence of PCOS accounts for 6-10\% of women's reproductive years $(27,28)$. PCOS is a primary cause of infertility and the growth of ovarian granulosa cells has been associated with the development of PCOS (29). In the present study, IOSE80 cells and human ovarian granulosa cell-like KGN cells were used and it was discovered that the expression levels of miR-206 were downregulated in $\mathrm{KGN}$ cells, which is consistent with a previous report (24). The results also suggested that the downregulated miR-206 expression levels may be associated with the occurrence and development of PCOS.

The present study further investigated the mechanisms of miR-206 in PCOS. It was previously reported that CCND2 was a target gene of miR-206 (30), and the present study validated this finding. In addition, miR-206 mimic significantly reduced CCND2 expression in KGN cells, indicating a negative regulatory mechanism was identified between miR-206 and its target gene CCND2. To determine whether miR-206 affected the viability of KGN cells by directly targeting CCND2, several experiments were performed in KGN cells following the overexpression of miR-206 or CCND2. The results of the MTT and flow cytometric analysis indicated that the overexpression of miR-206 reduced the cell viability and induced cell apoptosis in KGN cells, while these effects were reversed following the upregulation of CCND2. However, in the present study, the cell viability and apoptosis of cells was detected using an MTT assay and flow cytometric analysis, respectively; it is more reliable and convincing to detect cell viability and the levels of apoptosis by various experimental methods (such as TUNEL and BrdU assay), thus this is a limitation of the present study.

The caspase family serve an important role in the process of mediating cell apoptosis (31). Caspase-3, which belongs to the cell death protein 3 subfamily, is the crucial executor molecule that participates in numerous pathways of apoptosis signal transduction $(32,33)$. The present study analyzed caspase-3 activity and expression levels, and the results showed that miR-206 mimic significantly enhanced caspase- 3 activity, increased cleaved-caspase- 3 protein level, reduced pro-caspase- 3 protein level and enhanced the ratio of cleaved-caspase-3/pro-caspase-3, and all these changes were reversed by CCND2-plasmid co-transfection. The findings indicated that miR-206 and CCND2 may affect the viability of KGN cells via regulating caspase-3.

In conclusion, the findings of the present study suggested that miR-206 may serve a crucial role in PCOS through modulating the cell viability and apoptosis of ovarian granulosa cells. These findings indicated that miR-206 may be an effective target for the treatment of PCOS, which provides a novel opportunity for the development of clinical therapies for PCOS. However, the present study was only a preliminary study of the role of miR-206 in PCOS. In order to clarify the role and function of miR-206 in PCOS, further in-depth research is required. For example, the expression levels of miR-206 and its target gene CCND2 in ovarian tissue or granulosa cells of patients with PCOS needs to be clarified. Furthermore, the role of CCND2 in regulating the cell viability and apoptosis of ovarian granulosa cells should be determined. In addition, the effects of miR-206 and CCND2 in PCOS should be investigated in vivo; for instance, the association between the expression levels of miR-206 and CCND2 and the clinicopathological variables of patients with PCOS requires further analysis. Future research will aim to explore these topics in depth.

\section{Acknowledgements}

Not applicable.

\section{Funding}

The present study was supported by the Project of Study on Meiosis and Signal Transduction Pathway of Mouse Oocytes (grant no. LY20H040001).

\section{Availability of data and materials}

The datasets used and/or analyzed during the current study are available from the corresponding author on reasonable request.

\section{Authors' contributions}

JZ and XJ contributed to the conception and design of the study, data acquisition, analysis and interpretation, and drafted and critically revised the manuscript. ZS collected the data and performed statistical analysis. $\mathrm{ZZ}$ contributed to data collection and prepared the manuscript. All authors read and approved the final manuscript.

\section{Ethics approval and consent to participate}

Not applicable.

\section{Patient consent for publication}

Not applicable. 


\section{Competing interests}

The authors declare that they have no competing interests.

\section{References}

1. Macut D, Bjekić-Macut J, Rahelić D and Doknić M: Insulin and the polycystic ovary syndrome. Diabetes Res Clin Pract 130 163-170, 2017.

2. Franik S, Eltrop SM, Kremer JA, Kiesel L and Farquhar C: Aromatase inhibitors (letrozole) for subfertile women with polycystic ovary syndrome. Cochrane Database Syst Rev 5: CD010287, 2018

3. Öztürk A, Kucur SK, Seven A, Deveci E, Sencan H, Yilmaz O and Kiliç A: Temperament and character differences of patients with polycystic ovary syndrome. J Gynecol Obstet Hum Reprod 48: 255-259, 2019

4. Cooney LG and Dokras A: Beyond fertility: Polycystic ovary syndrome and long-term health. Fertil Steril 110: 794-809, 2018.

5. Kshetrimayum C, Sharma A, Mishra VV and Kumar S: Polycystic ovarian syndrome: Environmental/occupational, lifestyle factors; an overview. J Turk Ger Gynecol Assoc 20: 255-263, 2019.

6. Sathyapalan T, Shepherd J, Arnett C, Coady AM, Kilpatrick ES and Atkin SL: Atorvastatin increases 25 -hydroxy vitamin D concentrations in patients with polycystic ovary syndrome. Clin Chem 56: 1696-1700, 2020.

7. Li L, Zhang R, Zeng J, Ke H, Peng X, Huang L, Zhang H, Chen Z, Li TT, Tan Q, et al: Effectiveness and safety assessment of drospirenone/ethinyl estradiol tablet in treatment of PCOS patients: A single center, prospective, observational study. BMC Womens Health 20: 39, 2020.

8. Xu J, Bao X, Peng Z, Wang L, Du L, Niu W and Sun Y: Comprehensive analysis of genome-wide DNA methylation across human polycystic ovary syndrome ovary granulosa cell. Oncotarget 7: 27899-27909, 2016.

9. Lai Q, Xiang W, Li Q, Zhang H, Li Y, Zhu G, Xiong C and Jin L: Oxidative stress in granulosa cells contributes to poor oocyte quality and IVF-ET outcomes in women with polycystic ovary syndrome. Front Med 12: 518-524, 2018.

10. Lv X, He C, Huang C, Wang H, Hua G, Wang Z, Zhou J, Chen X, Ma B, Timm BK, et al: Timely expression and activation of YAP1 in granulosa cells is essential for ovarian follicle development FASEB J 33: 10049-10064, 2019.

11. Fan Y, Chang Y, Wei L, Chen J, Li J, Goldsmith S, Silber S and Liang X: Apoptosis of mural granulosa cells is increased in women with diminished ovarian reserve. Version 2. J Assist Reprod Genet 36: 1225-1235, 2019.

12. Fu X, He Y, Wang X, Peng D, Chen X, Li X and Wan Q: MicroRNA-16 promotes ovarian granulosa cell proliferation and suppresses apoptosis through targeting PDCD4 in polycystic ovarian syndrome. Cell Physiol Biochem 48: 670-682, 2018.

13. Zheng Q, Li Y, Zhang D, Cui X, Dai K, Yang Y, Liu S, Tan J and Yan Q: ANP promotes proliferation and inhibits apoptosis of ovarian granulosa cells by NPRA/PGRMC1/EGFR complex and improves ovary functions of PCOS rats. Cell Death Dis 8: e3145, 2017.

14. Li M, Zhao H, Zhao SG, Wei DM, Zhao YR, Huang T, Muhammad T, Yan L, Gao F, Li L, et al: The HMGA2-IMP2 pathway promotes granulosa cell proliferation in polycystic ovary syndrome. J Clin Endocrinol Metab 104: 1049-1059, 2019.

15. Wang J, Chen J and Sen S: MicroRNA as biomarkers and diagnostics. J Cell Physiol 231: 25-30, 2016.
16. Yanaihara $\mathrm{N}$ and Harris CC: MicroRNA involvement in human cancers. Clin Chem 59: 1811-1812, 2013.

17. Liao XB, Perez VA, Król M, Yeh CH and Yuan LQ: MicroRNA and cardiovascular disease. Biomed Res Int 2015: 734380, 2015.

18. Gong CL, Wang Y, Lyu Y, Sun JJ and Li GP: The effect and mechanism of microRNA199 in gynecological diseases. J Fam Plam Reprod H 33: 60-63, 2014.

19. Guo J, Sun M, Teng X and Xu L: MicroRNA-7-5p regulates the expression of TFF3 in inflammatory bowel disease. Mol Med Rep 16: 1200-1206, 2017.

20. Wang M, Sun J, Xu B, Chrusciel M, Gao J, Bazert M, Stelmaszewska J, Xu Y, Zhang H, Pawelczyk L, et al: Functional characterization of microRNA-27a-3p expression in human polycystic ovary syndrome. Endocrinology 159: 297-309, 2018.

21. Sørensen AE, Wissing ML, Englund AL and Dalgaard LT: MicroRNA species in follicular fluid associating with polycystic ovary syndrome and related intermediary phenotypes. J Clin Endocrinol Metab 101: 1579-1589, 2016.

22. Roth LW, McCallie B, Alvero R, Schoolcraft WB, Minjarez D and Katz-Jaffe MG: Altered microRNA and gene expression in the follicular fluid of women with polycystic ovary syndrome. J Assist Reprod Genet 31: 355-362, 2014.

23. Ilie IR and Georgescu CE: Polycystic ovary syndrome-epigenetic mechanisms and aberrant microRNA. Adv Clin Chem 71:25-45, 2015.

24. Díaz M, Bassols J, López-Bermejo A, de Zegher F and Ibáñez L: Low circulating levels of miR-451a in girls with polycystic ovary syndrome: Different effects of randomized treatments. J Clin Endocrinol Metab 105: 1-9, 2020.

25. Sun X, Su S, Zhang G, Zhang H and Yu X: MiR-204 suppresses cell proliferation and promotes apoptosis in ovarian granulosa cells via targeting TPT1 in polycystic ovary syndrome. Biochem Cell Biol 97: 554-562, 2019.

26. Livak KJ and Schmittgen TD: Analysis of relative gene expression data using real-time quantitative PCR and the 2(-Delta Delta C(T)) method. Methods 25: 402-408, 2001

27. Bahri Khomami M, Boyle JA, Tay CT, Vanky E, Teede HJ, Joham AE and Moran LJ: Polycystic ovary syndrome and adverse pregnancy outcomes: Current state of knowledge, challenges and potential implications for practice. Clin Endocrinol (Oxf) 88: 761-769, 2018.

28. Cui Y, Shi Y, Cui L, Han T, Gao X and Chen ZJ: Age-specific serum antimullerian hormone levels in women with and without polycystic ovary syndrome. Fertil Steril 102: 230-236, 2014.

29. Wei D, Xie J, Yin B, Hao H, Song X, Liu Q, Zhang C and Sun Y: Significantly lengthened telomere in granulosa cells from women with polycystic ovarian syndrome (PCOS). J Assist Reprod Genet 34: 861-866, 2017.

30. Chang L, Guo R, Yuan Z, Shi H and Zhang D: LncRNA HOTAIR regulates CCND1 and CCND2 expression by sponging miR-206 in ovarian cancer. Cell Physiol Biochem 49: 1289-1303, 2018.

31. Van Opdenbosch N and Lamkanfi M: Caspases in cell death, inflammation, and disease. Immunity 50: 1352-1364, 2019.

32. Pang X, Li K, Wei L, Huang Y, Su M, Wang L, Cao H and Chen T: IL-8 inhibits the apoptosis of MCF-7 human breast cancer cells by up-regulating Bcl-2 and down-regulating caspase-3. Xi Bao Yu Fen Zi Mian Yi Xue Za Zhi 31: 307-311, 2015 (In Chinese).

33. Zhang Y, Jiang Q, Wang N, Dai B, Chen Y and He L: Effects of taspine on proliferation and apoptosis by regulating caspase-3 expression and the ratio of $\mathrm{Bax} / \mathrm{Bcl}-2$ in A431 cells. Phytother Res 25: 357-364, 2011.

This work is licensed under a Creative Commons Attribution-NonCommercial-NoDerivatives 4.0 International (CC BY-NC-ND 4.0) License. 